\title{
Learning virtually meaningless metaphors under different instructional conditions
}

\author{
RICHARD DOLINSKY \\ University of Toledo, Toledo, Ohio 43606 \\ and \\ KAREN M. ZABRUCKY \\ Clemson University, Clemson, South Carolina 29631
}

\begin{abstract}
It has been proposed that "the distinction between metaphor and simple deviance involves the intention ... to be metaphorical" (Matthews, 1971, p. 417). This supposition was tested by comparing sentence recall in metaphors rated very low in meaningfulness with similarly rated nonmetaphors and nonmetaphors rated highly meaningful. Two instructional conditions were evaluated, literary source instructions, which intended to communicate that the sentences made sense, and nonliterary instructions, which did not have this intention. Generally, metaphors were learned as well as meaningful nonmetaphors and both were learned better than meaningless nonmetaphors. It was suggested that the comparison stated in metaphors is adequate to communicate intention regardless of a particular instructional set.
\end{abstract}

In metaphor, a comparison is made, presumably one that is meaningful to the listener, but one that clearly is not to be taken as a statement of fact. To say that "because of too much summer sun, her hair turned to straw" is to state something that is nonfactual; no one believes that her hair actually became transmuted into straw. But this metaphor is meaningful because the comparison it makes tells us something about the effects of the summer sun on somebody's hair. The meaning, however, is less obvious than a direct statement of fact.

We may argue that when a listener or reader encounters a metaphor, he expects it to carry some coherent meaning and, further, that he expects to do some abstracting in order to understand the intent of the comparison. It is this expansion of the semantic frame of reference that makes metaphor both a useful literary tool and a psychological phenomenon of importance.

Apparently, specific syntactic surface structure is not a relevant criterion for metaphor (cf. Loewenberg, 1975, p. 315); therefore, the knowledge of how metaphors operate is dependent on other factors. While it is clear that some bizarre sentences might carry very little meaning, it also has been suggested that "a hearer will try to interpret or at least account for a speaker's utterances. He will often accept and make sense out of sentences that appear odd or contradictory when presented to him." (Bendix, 1971, p. 406). That is, a person might tolerate more ambiguity in a metaphor. Nonsensical or deviant sentences that sound like metaphors,

Requests for reprints may be sent to Karen M. Zabrucky, Department of Psychology, Clemson University, Clemson, South Carolina 29631. in that they make comparisons, might be dismissed less readily than nonsensical, or deviant, nonmetaphors.

Then what makes an utterance a metaphor? One suggestion is that the definition lies in the situation. Matthews (1971) notes that "given the proper context (linguistic and extralinguistic) almost any deviant sentence can be interpreted as metaphorical" and that "the distinction between metaphor and simple deviance involves the intention ... to be metaphorical." (pp. 416417). If this shared intention between the speaker and listener is a major factor in metaphor, then understanding of the metaphor includes more than the linguistic meaning of the sentence itself. That is, the issue seems to take on the trappings of a social contract. Everyone involved believes the communication makes sense.

Ample evidence exists to show that verbal material is more easily recalled when it is meaningful (Marks \& Miller, 1964). If listeners believe that the metaphors they hear are meaningful, we might expect that these metaphors would be more easily recalled than other sentences that are not believed to carry meaning. Following this assumption, it should be possible to influence the recall of metaphors as a function of the learner's conceptions about the meaningfulness of the sentences they are considering.

In the following experiments, we investigated how metaphors were learned when random nouns were inserted into a grammatical sentence frame. We tried to influence the recall of the resulting, virtually meaningless, sentences by manipulating the instructions to the learners. If the instructions effectively gave the intent that the sentences were meaningful, that they were from literary sources, for example, it was predicted recall 
would be superior to a situation in which this intent was not communicated. In fact, we thought it not unlikely that these meaningful, "literary" instructions would lead to recall performance similar to that elicited by meaningful nonmetaphorical sentences that are simple statements of fact.

\section{EXPERIMENTS 1 AND 2}

These two experiments are most effectively treated together, the distinction between them being a temporal one. Experiment 2 was conducted at the completion of Experiment 1.

\section{Method}

Materials. A group of metaphors was constructed by using a grammatical frame typical for comparisons: The $\_$is the of the . Nouns were inserted at random into the slots in the sentence, so that the final outcome was grammatical but meaningless (e.g., "The drum is the castle of the mop."). A second group of sentences, not forming comparisons and considered to be meaningless and nonmetaphorical, was constructed in a similar manner (e.g., "The pipe and prairie are on the needle."). Finally, there was a third group of sentences presumed to be meaningful nonmetaphorical statements that used the same sentence frame as the other nonmetaphorical sentences.

The same nouns were used in all three groups, there was no obvious associative relationship between nouns in the same sentence, and all had high imagery ratings (Paivio, Yuille, \& Madigan, 1968). There were nine sentences in each of the three groups.

Ratings of meaningfulness. While it seemed apparent from casual inspection that the "meaningless" metaphors and nonmetaphors were clearly bizarre, this observation was corroborated by 17 introductory psychology students who rated all materials on a 5-point meaningfulness scale ( 1 for least and 5 for most). Mean ratings were $1.33,1.30$, and 4.55 for the meaningless nonmetaphors, meaningless metaphors, and meaningful nonmetaphors, respectively. The meaningful group differed significantly from the other two $[\mathrm{t}(16)=49.57$ and $49.83, \mathrm{p}<.01]$, but the former two groups did not differ $[t(16)=.44]$.

Procedure. The design for Experiment 1 was a betweensubjects 2 by 3 factorial with 30 subjects in each group. There were three types of sentence materials: meaningful nonmetaphors, meaningless nonmetaphors, and meaningless metaphors. There were also two types of instructional sets: standard and "literary source." Subjects in the three standard groups were given typical learning instructions, namely, to try to learn the sentences. Subjects in the literary source groups were told: "These sentences have all been taken from literary sources and have been adapted for use in this experiment ..... In context they are meaningful but will appear out of context in this experiment so treat them as normal meaningful sentences." Obviously, the literary source instructions tried to present a strong case for the semantic coherence of the materials.

In a pilot study using a similar design, subjects were told that the sentences in both the standard and literary source metaphor conditions were metaphors, a metaphor was defined for them, and it was obvious from the sentence frames that a comparison was being proposed. In that study, we found that meaningless metaphors were learned more quickly than meaningless nonmetaphors and as well as meaningful nonmetaphors. Whether our instructions effectively implied a literary source or not, we felt that the knowledge that a sentence was intended to be both a metaphor and meaningful confounded the situation and might have biased the results toward superior learning in the metaphor conditions. Thus, in the present study, the term metaphor was not used. For both standard and literary source conditions, all materials were referred to as sentences.
Subjects in all six groups received three lists with three sentences in each. Each list was presented for three trials with written recall after each trial. A probe technique was used, with one noun in each sentence used as the recall probe. Each of the three nouns in a sentence was used equally often as a probe. The conditions were run in introductory psychology class groups of various numbers, and, for the final analyses, individual records were randomly discarded until each condition was composed of 30 individuals. The sentences were tape-recorded with normal intonation and tempo (approximately $2 \mathrm{sec}$ each), and there was an 8 -sec interval between sentences. The probed recall allowed $15 \mathrm{sec}$ for each sentence, and there was a 30 -sec intertrial interval.

Experiment 2 compared only the meaningless nonmetaphors used in Experiment 1, but now the sentences were referred to as "metaphors" in both the standard and literary source conditions. A metaphor was defined as "a sentence that makes a comparison without using 'like' or 'as,'" and an example was given. The aim was to determine whether the metaphor name cue could affect recall in the absence of a sentence frame that indicated a comparison. Thirty introductory psychology students served as subjects in each of the two conditions.

\section{Results}

The combined results, along with the results for the individual lists, are presented in Table 1 for both Experiments 1 and 2 . Only verbatim sentence recall to the noun probe was considered correct. For Experiment 1, the combined results indicate a significant effect between sentence types $[F(2,174)=18.44, p<.01]$. Meaningful nonmetaphors were recalled significantly better than metaphors, and these were significantly superior to meaningless nonmetaphors ( $\mathrm{p}<.01$ for all comparisons). The effect of instructional set was not significant $(\mathrm{F}<1)$. There was improvement in recall as lists progressed $[F(2,348)=45.15, \mathrm{p}<.01]$ and a significant trials effect $[F(2,348)=437.55, p<.01]$. None of the interactions was significant after the GreenhouseGeisser (1959) adjustment, with the exception of that among lists, instructions, and trials $[\mathrm{F}(8,696)=6.41$, $\mathrm{p}<.05]$.

The individual lists show effects similar to the combined results. Differences between sentence types were in the same order $[F(2,174)=21.22,14.75$, and 4.30 for Lists 1-3, respectively]. The effect was significant at the .01 level for the first two lists and at the .05 level for the third. In distinction to the combined results, however, the meaningful nonmetaphors and metaphors were not significantly different on any of the individual lists, although both differed from the meaningless nonmetaphors on Lists 1 and 2. On List 3, meaningless

Table 1

Proportion of Sentences Correct in Experiments 1 and 2 for Different Lists and Instructional Sets

\begin{tabular}{|c|c|c|c|c|c|c|c|c|}
\hline & \multicolumn{2}{|c|}{ List 1} & \multicolumn{2}{|c|}{ List 2} & \multicolumn{2}{|c|}{ List 3} & \multicolumn{2}{|c|}{$\mathrm{C}$} \\
\hline & $S$ & $\mathrm{~L}$ & $\mathbf{S}$ & L & $S$ & $\mathbf{L}$ & $\mathbf{S}$ & $\mathbf{L}$ \\
\hline Meaningful Sentences & .54 & .47 & .66 & .68 & .66 & .71 & .62 & .62 \\
\hline Metaphors & .53 & .46 & .59 & .60 & .61 & .59 & .58 & .55 \\
\hline Meaningless Sentences* & .21 & .27 & .39 & .41 & .51 & .56 & .37 & .42 \\
\hline Meaningless Sentences $\nmid$ & .23 & .25 & .34 & .32 & .49 & .40 & .35 & .32 \\
\hline
\end{tabular}

Note- $S=$ standard instruction set, $L=$ literary instruction set; $C=$ Lists $1-3$ combined. $\quad{ }^{*}$ Experiment $1 . \quad$ tExperiment 2. 
metaphors and nonmetaphors did not differ. The fact that the differences were reduced in List 3 probably reflects the effects of practice. At any rate, the general findings seem clear; meaningless metaphors function like meaningful nonmetaphors.

The instruction difference was not significant for any list $(\mathrm{F}<1)$; trials was significant throughout, and the only interaction worth noting was that between sentence type and trials on Lists 1 and 3. There was a greater increase in recall over trials for the meaningless nonmetaphors than for the other two types, both of which began at higher levels on the first trial.

The findings for Exepriment 2 indicated a nonsignificant difference between the two conditions $[\mathrm{t}(58)=$ .57] and no significant differences when compared with the meaningless nonmetaphors in Experiment 1. With these sentences, which did not present a comparison in the sentence frame, it seems as if calling something a metaphor and defining it is no better than just referring to it as a sentence.

\section{Discussion}

Why, then, were the metaphors learned better than similarly rated nonmetaphors? In a further attempt to explain the findings, individuals from the three literary source conditions in Experiment 1 were asked several questions following their participation: (1) what literary sources they thought the sentences were drawn from, (2) how they thought the sentences were adapted for the experiment, and (3) whether the sentences were meaningful.

For the first question, there was some uniformity among the individuals receiving the three sentence types. About $38 \%$ had no idea from which source the materials were drawn; $13 \%$ indicated children's books, and another $23 \%$ novels. Poetry was mentioned $27 \%$ of the time for the metaphors and $20 \%$ for the meaningless nonmetaphors, but never for the meaningful sentences.

For the second question, more individuals from the meaningful sentence condition indicated they had no idea how the sentences were adapted ( $40 \%$ vs. about $20 \%$ for the meaningless conditions). For the meaningless conditions, the dominant explanation was that the words had been switched around within the sentence $(43 \%$ and $30 \%$ for the nonmetaphors and metaphors, respectively).

For the third question, $57 \%$ in the meaningful condition thought at least some of the sentences were meaningful. This figure was $23 \%$ in the metaphor condition, but only $3 \%$ (one person) in the meaningless nonmetaphor group. While the low frequencies restrict definite conclusions, they suggest that after the learning task, learners in the metaphor condition (at least those receiving literary source instructions) were more likely to consider meaningful interpretations than were those in the nonmetaphor condition.

\section{CONCLUSION}

In summary, the present data indicate that metaphors rated as virtually meaningless are learned better than similarly rated nonmetaphors and as well, or almost as well, as meaningfully rated nonmetaphors. Specific instructions as to the source of the sentences was not found to be relevant. On the surface, the insensitivity of instructional set would appear to deny the intention hypothesis, the notion that a metaphor is accepted as such because of an agreement between speaker and listener to use language in a nonfactual way. However, it is not necessary to abandon this hypothesis.

The findings indicate that there is something about the metaphors that makes them easier to learn than the meaningless nonmetaphors and, apparently, it is something in the material itself, rather than the specific instructions. What seems a reasonable possibility is that the metaphorical sentence frames used in these experiments indicate that a comparison is intended even though the nature of the comparison might be obscure. To say that "the flower is the bulldozer of the moon" compares the flower's function on the moon to that of a bulldozer. Although the relationship is not obvious, it is at least obvious that some relationship, whatever it might be, is intended. In this sense, then, the relevance of the intention hypothesis is preserved.

If we assume that the comparison in the metaphors results in attributing more meaning to them than to the meaningless nonmetaphors, then why were both types of sentences similarly rated as meaningless? There are several reasons. The raters faced a task that could be accomplished in a cursory manner: simply to read a sentence and make a snap rating. There was no need to learn anything, to develop any storage and retrieval strategies, or to organize in any way. Probably, the raters spend no more than a few seconds on each sentence. The implication, then, is that the sentence either made sense immediately or it did not. The rating was almost automatic.

In addition, material from all three sentence types was presented randomly to the raters, and they quite rapidly noticed that the sentences fell into two categories: those that made sense and those that did not. Because all three sentence types were included, the frame of reference for the ratings was different from that in the learning task, in which only one sentence condition was presented to each individual. In this condition, finer discrimination of meaningfulness might have been made. Trying to find meaning in the sentences might, itself, be used as a learning strategy, similar to the "contextualist" interpretations given to word list learning (Jenkins, 1974). And, for the metaphors, the learner's assumption that a nonfactual comparison was also intended might have further tipped the subjective scale, resulting in more effective learning.

Finally, the metaphors we have used obviously are only a very small subset of the different ways metaphors can be expressed. In other sentences, the awareness that some comparison is intended might be much less apparent. It remains, then, to determine whether other types of metaphors will be learned in a manner similar to those investigated in these experiments.

\section{REFERENCES}

Bendix, E. H. The data of semantic description. In D. D. Steinberg \& L. A. Jakobovits (Eds.), Semantics. Cambridge: Cambridge University Press, 1971.

Greenhouse, S. W., \& Geisser, S. On the methods in the arialysis of profile data. Psychometrika, 1959, 24, 95-112.

Jenkins, J. J. Remember that old theory of memory? Well, forget it! American Psychologist, 1974, 29, 785-795.

Loewenbera, I. Identifying metaphors. Foundations of Language, 1975, 12, 315-338.

Marks, L. E., \& Mille R, G. A. The role of semantic and syntactic constraints in the memorization of English sentences. Journal of Verbal Learning and Verbal Behavior, 1964, 3, 1-5.

Matthews, R. Concerning a "linguistic theory" of metaphor. Foundations of Language, 1971, 7, 413-425.

Paivio, A., Yuille, J. C., \& Madigan, S. A. Concreteness, imagery, and meaningfulness values for 925 nouns. Journal of Experimental Psychology Monograph Supplement, 1968, 76(Whole No. 1, Pt. 2).

(Received for publication February 28, 1983.) 